\title{
The Kalai-Smorodinsky solution with loss aversion
}

Citation for published version (APA):

Driesen, B. W. I., Perea ý Monsuwé, A., \& Peters, H. J. M. (2009). The Kalai-Smorodinsky solution with loss aversion. METEOR, Maastricht University School of Business and Economics. METEOR Research Memorandum No. 030 https://doi.org/10.26481/umamet.2009030

Document status and date:

Published: 01/01/2009

DOI:

10.26481/umamet.2009030

Document Version:

Publisher's PDF, also known as Version of record

\section{Please check the document version of this publication:}

- A submitted manuscript is the version of the article upon submission and before peer-review. There can be important differences between the submitted version and the official published version of record.

People interested in the research are advised to contact the author for the final version of the publication, or visit the DOI to the publisher's website.

- The final author version and the galley proof are versions of the publication after peer review.

- The final published version features the final layout of the paper including the volume, issue and page numbers.

Link to publication

\footnotetext{
General rights rights.

- You may freely distribute the URL identifying the publication in the public portal. please follow below link for the End User Agreement:

www.umlib.nl/taverne-license

Take down policy

If you believe that this document breaches copyright please contact us at:

repository@maastrichtuniversity.nl

providing details and we will investigate your claim.
}

Copyright and moral rights for the publications made accessible in the public portal are retained by the authors and/or other copyright owners and it is a condition of accessing publications that users recognise and abide by the legal requirements associated with these

- Users may download and print one copy of any publication from the public portal for the purpose of private study or research.

- You may not further distribute the material or use it for any profit-making activity or commercial gain

If the publication is distributed under the terms of Article $25 \mathrm{fa}$ of the Dutch Copyright Act, indicated by the "Taverne" license above, 


\section{Maastricht University}

Bram Driesen, Andrés Perea, Hans Peters

The Kalai-Smorodinsky Solution with Loss Aversion

$\mathrm{RM} / 09 / 030$

\section{METEOR}

Faculty of Economics and Business Administration Maastricht Research School of Economics

of Technology and Organization

P.O. Box 616

NL - 6200 MD Maastricht

The Netherlands 


\title{
The Kalai-Smorodinsky Solution with Loss Aversion
}

\author{
Bram Driesen* Andrés Perea* Hans Peters*
}

This version, July 2009

\begin{abstract}
We consider bargaining games under the assumption that bargainers are loss averse, i.e. experience disutility from obtaining an outcome lower than some reference point. We follow the approach of Shalev (2002) by imposing the self-supporting condition on a solution. Given a bargaining game, we say outcome $z$ is self-supporting under a given bargaining solution, whenever transforming the game using outcome $z$ as reference point, yields a transformed game in which the solution is $z$.

We show that $n$-player bargaining games have a unique self-supporting outcome under the Kalai-Smorodinsky (KS) solution. We define a bargaining solution, giving exactly this outcome, and characterize it by the standard axioms of Scale Invariance [SI], Individual Monotonicity [IM], and Strong Individual Rationality [SIR], and a novel axiom called Proportional Concession Invariance [PCI]. A bargaining solution satisfies PCI if moving the utopia point in the direction of the solution outcome, does not change this outcome.
\end{abstract}

\section{Introduction}

In his seminal paper, Nash (1950) defined the 'bargaining problem' as a proxy for real life bargaining situations. His model is described by two players attempting to find agreement on a certain feasible outcome. They are motivated by the fact that failure to cooperate results in an unfavorable outcome for all. Nash himself defined and characterized a solution for this problem, the well known Nash bargaining solution. A wide range of other solutions have been formulated since. One of the most prominent alternatives to the Nash bargaining solution is the Kalai-Smorodinsky solution, defined by Raiffa (1953) and characterized by Kalai and Smorodinsky (1975).

* Department of Quantitative Economics, University of Maastricht, P.O. Box 616, 6200 MD Maastricht, The Netherlands. Telephone: +31-43-3883835. Telefax: +31-43-3884874. Email adresses: B.Driesen@maastrichtuniversity.nl, A.Perea@maastrichtuniversity.nl, H.Peters@maastrichtuniversity.nl. 
In economics - and bargaining is no exception - risk attitudes tend to play a pervasive role on how agents behave. In the bargaining literature, much attention has been paid to the influence of risk attitudes, and in particular risk aversion, on the outcomes players finally agree on according to certain bargaining solutions. Several studies, among which Kannai (1977), Kihlstrom, Roth, and Schmeidler (1981), and Roth (1985), find that the Nash bargaining solution favors the less risk averse bargainer. Kihlstrom, Roth and Schmeidler (1981) find similar results for the Kalai-Smorodinsky solution. Köbberling and Peters (2003) also study the effect of risk aversion on the Kalai-Smorodinsky solution, but distinguish between probabilistic risk aversion and utility risk aversion. They find it is an advantage to have a more utility risk averse opponent, or a less probabilistically risk averse opponent.

In the present article we investigate the Kalai-Smorodinsky solution under a related behavioral phenomenon, called loss aversion. Loss aversion was introduced by Kahneman and Tversky $(1979,1984)$. It is based on the premise that economic agents derive utility from changes in wealth in comparison to some reference point, and that changes for the worse - i.e. losses - carry more weight than equivalent changes for the better.

To introduce this concept in the bargaining problem, we follow the approach of Shalev (2002). Each bargainer's preference is represented by a von NeumannMorgenstern utility function, a nonnegative loss aversion coefficient, and a reference point; if a player's utility level is below his reference point, then he experiences a disutility equal to the size of his incurred loss, inflated by the loss aversion coefficient. Thus, in a bargaining context, incorporating the players' loss aversion is equivalent to applying a particular transformation to the bargaining problem. Reference points are subsequently endogenized by imposing the self-supporting condition. An outcome $z$ is said to be self-supporting under a given solution, whenever transforming the bargaining game using outcome $z$ as reference point, yields a game of which the solution is $z$. We may interpret a bargainer's reference point as the expectation or the aspiration of what utility payoff that bargainer may realize given a certain bargaining solution. The selfsupporting condition then imposes that the bargaining solution assigns to each player exactly the (initially) aspired utility level, and as a consequence, that no losses are incurred.

The first purpose of this article is to show that $n$-player bargaining games, $n \geq 2$, have exactly one outcome that is self-supporting under the KalaiSmorodinsky solution ${ }^{1}$. Kalai and Smorodinsky (1975) defined their solution on two-player bargaining games. In view of Roth's (1979) result that it is not generally defined on all $n$-player bargaining games, we consider the subclass of all $n$-player bargaining games, defined by Peters and Tijs (1984), which excludes the problematic cases. On these games we define a class of asymmetric $n$-person Kalai-Smorodinsky solutions. Consider an outcome in the Pareto set and the line segments respectively connecting that outcome to the disagreement

\footnotetext{
${ }^{1}$ This was already remarked - without proof - by Shalev (2002) for the case of two-player bargaining games.
} 
point and the utopia point. For any two players we may then project these line segments into the plane. Our solution is defined by the unique outcome in the Pareto set such that for any two players, the slopes of these projected line segments satisfy a given proportion. We establish that for each loss aversion profile, there is a single bargaining solution in our class that yields the associated self-supporting outcome. This implies uniqueness of a self-supporting outcome under the Kalai-smorodinsky solution for $n$-player bargaining games. Moreover, the bargaining solution we define provides an easy method of finding this outcome.

We next provide an axiomatic characterization of this class of bargaining solutions. In particular, we show that it is fully defined by the standard properties Strong Individual Rationality, Scale Invariance, and Individual Monotonicity, and a new axiom named Proportional Concession Invariance [PCI]. The axiom of PCI says that if players make concessions with respect to their utopia values in such a way that the new utopia point is on the line segment connecting the solution outcome and the original utopia point, then the solution outcome is left unchanged.

The article proceeds as follows. Section 2 introduces the notation, and in Section 3 we show how loss aversion is incorporated in the bargaining problem. Section 4 describes the concept of monotonic curves, their associated bargaining solutions, and defines the Kalai-Smorodinsky solution as a special case. In Section 5 we define a class of bargaining solutions, and we show that every solution in this class is the self-supporting outcome under the Kalai-Smorodinsky solution for some loss aversion profile. Section 6 contains the axiomatic characterization of this class of bargaining solutions. We conclude in Section 7 .

All claims made in the text are proven in the Appendix.

\section{Preliminaries}

The set of players or bargainers is denoted $N:=\{1, \ldots, n\}$, with $n \geq 2$. For $x, y \in \mathbb{R}^{N}$ we say $x \geq y$ if $x_{i} \geq y_{i}$ for all $i \in N$, and $x>y$ if $x_{i}>y_{i}$ for all $i \in N$. The relations ' $\leq$ ' and ' $<$ ' are defined similarly. We define $\mathbb{R}_{+}^{N}:=\{z \in$ $\left.\mathbb{R}^{N} \mid z \geq 0\right\}$ and $\mathbb{R}_{++}^{N}:=\left\{z \in \mathbb{R}^{N} \mid z>0\right\}$. For $x, y \in \mathbb{R}^{N}$ we denote by $x y$ the vector $\left(x_{1} y_{1}, \ldots, x_{n} y_{n}\right)$, and for $S \subseteq \mathbb{R}^{N}$ we define $x S:=\left\{x y \in \mathbb{R}^{N} \mid y \in S\right\}$. Similarly, we denote $\left(x_{1}+y_{1}, \ldots, x_{n}+y_{n}\right)$ as $x+y$, and the set $\{x+z \mid z \in S\}$ as $x+S$. The vector in $\mathbb{R}^{N}$ that has $i$-th coordinate equal to 1 and all other coordinates equal to 0 is denoted $e^{i}$. The vector $e^{M}, M \subseteq N$ and $M \neq \emptyset$, has all coordinates $i \in M$ equal to 1 , and all coordinates $i \notin M$ equal to 0 . For $x \in \mathbb{R}_{++}^{N}$, we denote $\left(\frac{1}{x_{1}}, \ldots, \frac{1}{x_{n}}\right)$ as $x^{-1}$.

A bargaining problem for $N$ is defined as a pair $(S, d)$ where

- $S \subset \mathbb{R}^{N}$ is non-empty, closed, convex, and comprehensive,

- $d \in S$

- there exists a $z \in S$ such that $z>d$, and 
- $S_{d}:=\{z \in S \mid z \geq d\}$ is bounded.

A set $S$ is comprehensive if $x \in S$ and $x \geq y$ together imply $y \in S$. In a bargaining context, this assumption can be interpreted as free disposal of utility in the sense that any player can choose a lower utility payoff without it leading to an infeasible outcome. Bargainers seek agreement on an outcome $z$ in $S$ where each player $i \in N$ obtains utility $z_{i}$. In case no agreement is reached the disagreement outcome $d$ results.

The set of all bargaining problems is denoted $B^{N}$. For $(S, d) \in B^{N}$ and each $i \in N$, we define

$$
u_{i}(S, d):=\max \left\{z_{i} \mid z \in S_{d}\right\} .
$$

This represents the highest possible utility payoff bargainer $i$ can attain in the bargaining problem $(S, d)$, given that no bargainer $j \in N, j \neq i$, obtains a utility payoff lower than $d_{j}$. The vector $u(S, d):=\left(u_{1}(S, d), \ldots, u_{n}(S, d)\right)$ is termed the utopia point of $(S, d)$. For all $(S, d) \in B^{N}$ we define the Pareto set of $(S, d)$ as

$$
P(S):=\left\{z \in S \mid \text { for all } x \in \mathbb{R}^{N} \text { if } x \geq z \text { and } x \neq z \text {, then } x \notin S\right\} .
$$

A bargaining solution or in short, a solution, is a map $\varphi: B^{N} \rightarrow \mathbb{R}^{N}$ that assigns to any bargaining problem $(S, d) \in B^{N}$ a single point $\varphi(S, d) \in S$.

\section{Bargaining with Loss Aversion}

Shalev $(2000,2002)$ introduced a transformation that models bargainers' loss aversion. Each bargainer $i \in N$ has a non-negative loss aversion coefficient $\lambda_{i}$ and a reference point $r_{i}$. We denote the vector $\left(\lambda_{1}, \ldots, \lambda_{n}\right) \in \mathbb{R}_{+}^{N}$ by $\lambda$. Similarly, $r:=\left(r_{1}, \ldots, r_{n}\right) \in \mathbb{R}^{N}$. Each bargainer $i$ evaluates a utility payoff $z_{i} \in \mathbb{R}$ by the transformation $w_{i}$, defined as

$$
w_{i}\left(z_{i}, \lambda_{i}, r_{i}\right):= \begin{cases}z_{i} & \text { if } z_{i} \geq r_{i} \\ z_{i}-\lambda_{i}\left(r_{i}-z_{i}\right) & \text { if } z_{i}<r_{i} .\end{cases}
$$

Thus, a bargainer $i$ who incurs a loss - i.e. obtains a utility payoff $z_{i}$ below his reference point $r_{i}$, experiences a disutility that is equal to his loss $r_{i}-z_{i}$, inflated by the loss aversion coefficient $\lambda_{i}$. Payoffs above the reference point are left unchanged. Note that we may also write

$$
w_{i}\left(z_{i}, \lambda_{i}, r_{i}\right)=\left(1+\lambda_{i}\right) z_{i}-\lambda_{i} \max \left\{r_{i}, z_{i}\right\} .
$$

For utility outcomes $z \in \mathbb{R}^{N}$ we define

$$
w(z, \lambda, r):=\left(w_{1}\left(z_{1}, \lambda_{1}, r_{1}\right), \ldots, w_{n}\left(z_{n}, \lambda_{n}, r_{n}\right)\right) .
$$

For sets $T \subset \mathbb{R}^{N}$ we write $w(T, \lambda, r):=\{w(z, \lambda, r) \mid z \in T\}$. Henceforth, the transformation $w: \mathbb{R}^{N} \times \mathbb{R}_{+}^{N} \times \mathbb{R}^{N} \rightarrow \mathbb{R}^{N}$ is referred to as the Shalev transformation. 
For bargaining problems $(S, d) \in B^{N}$ we define

$$
w((S, d), \lambda, r):=(w(S, \lambda, r), w(d, \lambda, r)) .
$$

We then have the following.

Lemma 3.1 Let $(S, d) \in B^{N}, \lambda \in \mathbb{R}_{+}^{N}$, and $r \in \mathbb{R}^{N}$. Then $w((S, d), \lambda, r) \in B^{N}$.

Let $\varphi: B^{N} \rightarrow \mathbb{R}^{N}$ be some bargaining solution, and let $(S, d)$ be some problem in $B^{N}$. Then by Lemma 3.1, $\varphi(w(S, d), \lambda, r)$ is well-defined for all $\lambda \in \mathbb{R}_{+}^{N}$ and $r \in \mathbb{R}^{N}$. A point $z \in S$ is called a self-supporting outcome under $\varphi$ if $z=\varphi(w((S, d), \lambda, z))$. The set of all such points is defined as

$$
\operatorname{Self}^{\varphi}((S, d), \lambda):=\{z \in S \mid z=\varphi(w((S, d), \lambda, z))\} .
$$

Then Self $\varphi: B^{N} \times \mathbb{R}_{+}^{N} \rightarrow \mathbb{R}^{N}$ is a correspondence that assigns to each bargaining problem $(S, d) \in B^{N}$ and loss aversion profile $\lambda \geq 0$ a possibly empty subset of $S$.

For two-player bargaining games $(S, d)$, Shalev (2002) characterized the set of self-supporting outcomes under the Nash bargaining solution. Specifically, he showed that it is a closed, connected subset of the Pareto set $P(S)$.

\section{The Kalai-Smorodinsky Solution}

Raiffa (1953), and Kalai and Smorodinsky (1975) defined and characterized a solution - the Kalai-Smorodinsky solution (KS) - for bargaining problems in $B^{\{1,2\}}$. It was shown by Roth (1979) that the $n$-player extension of the KS solution, $n>2$, is not defined on all bargaining problems in $B^{N}$. Therefore, Peters and Tijs (1984) introduced the rather large subclass $I^{N}$ of bargaining problems in $B^{N}$ for which this non-existence result does not hold. Consider the following condition.

For all $x \in S, x \geq d$, and $i \in N$ we have:

$x \notin P(S)$ and $x_{i}<u_{i}(S, d) \Rightarrow$ there is an $\varepsilon>0$ such that $x+\varepsilon e^{i} \in S$

Then

$$
I^{N}:=\left\{(S, d) \in B^{N} \mid(S, d) \text { satifies condition }(1)\right\} .
$$

Condition (1) says that if a feasible outcome $x$ is not Pareto optimal, then for any bargainer who does not get his utopia payoff there is a feasible outcome that this bargainer strictly prefers over $x$. The class of bargaining problems $(S, 0) \in I^{N}$ is denoted $I_{0}^{N}$; for bargaining problems in $I_{0}^{N}$ we henceforth omit the disagreement point, i.e. we denote $(S, 0) \in I_{0}^{N}$ as $S$.

Peters and Tijs (1984) defined the $n$-player extension of the KS solution by making use of monotonic curves. Specifically, a monotonic curve for $N$ is a map

$$
\vartheta:[1, n] \rightarrow\left\{x \in \mathbb{R}_{+}^{n} \mid x_{i} \leq 1 \text { for all } i \in N, \text { and } 1 \leq \sum_{i \in N} x_{i}\right\},
$$


such that for all $1 \leq s \leq t \leq n$ we have $\vartheta(s) \leq \vartheta(t)$ and $\sum_{i \in N} \vartheta_{i}(s)=s$. The set of all monotonic curves for $N$ is denoted $\Theta^{N}$.

Lemma 4.1 (Peters and Tijs, 1984) For each $\vartheta \in \Theta^{N}$ and $S \in I_{0}^{N}$ with $u(S)=e^{N}$, the set

$$
P(S) \cap\{\vartheta(t) \mid t \in[1, n]\}
$$

contains exactly one point.

Let $\vartheta$ be some monotonic curve in $\Theta^{N}$. In view of Lemma 4.1 we can define $\rho^{\vartheta}: I^{N} \rightarrow \mathbb{R}^{N}$, the solution associated with $\vartheta$. Let $S \in I_{0}^{N} ;$ if $u(S)=e^{N}$, then

$$
\left\{\rho^{\vartheta}(S)\right\}:=P(S) \cap\{\vartheta(t) \mid t \in[1, n]\},
$$

and if $u(S)=\beta$, then $\rho^{\vartheta}(S):=\beta \rho^{\vartheta}\left(\beta^{-1} S\right)$. For $(S, d) \in I^{N}$, we define $\rho^{\vartheta}(S, d):=d+\rho^{\vartheta}(S-d)$. The class of all solutions associated with a monotonic curve in $\Theta^{N}$ is referred to as the class of individually monotonic bargaining solutions. The KS solution is an element of this class. Specifically, it is defined as

$$
\rho^{\hat{\vartheta}} \text { where } \hat{\vartheta}(t):=\frac{t e^{N}}{n} \text { for } t \in[1, n] .
$$

Observe that $\hat{\vartheta}$ defines a straight line in $\mathbb{R}^{N}$, which for bargaining games $S \in I_{0}^{N}$ where $u(S)=e^{N}$, is concurrent with the line connecting the disagreement point 0 and the utopia point $e^{N}$. For general bargaining problems $(S, d) \in I^{N}$, the KS solution is the intersection of the Pareto set $P(S)$ and the straight line that connects the disagreement point $d$ and the utopia point $u(S, d)$. To ease notation, we also refer to $\rho^{\hat{\vartheta}}$ as $K S$, i.e. $K S \equiv \rho^{\hat{\vartheta}}$.

\section{The Solution Class $\mathcal{D}^{N}$}

In this section we show that self-supporting outcomes under the KS solution are well-defined, and that each game in $I^{N}$ has exactly one such outcome. Peters and Tijs (1984) show that $I^{\{1,2\}}=B^{\{1,2\}}$, which implies that our result generalizes Shalev's (2002) remark about the uniqueness of a self-supporting outcome under the KS solution for two-player bargaining games. Furthermore, we introduce a class $\mathcal{D}^{N}$ of bargaining solutions on $I^{N}$, where for any $\lambda \in \mathbb{R}_{+}^{N}$ there is a single $\varphi \in \mathcal{D}^{N}$ such that $\varphi(S, d)$ is the unique self-supporting outcome of the game $(S, d)$ under the KS solution.

Observe that from Lemma 3.1 and the fact that the Shalev transformation preserves the ordering of payoffs, we obtain that $(S, d) \in I^{N}$ implies $w((S, d), \lambda, r) \in I^{N}$ for all $\lambda \in \mathbb{R}_{+}^{N}$ and $r \in \mathbb{R}^{N}$. Therefore, Self ${ }^{K S}((S, d), \lambda)$, the set of self-supporting outcomes under the KS solution, is well-defined.

We now introduce the class $\mathcal{D}^{N}$ of bargaining solutions. Denote by $\bar{N}$ the player set without player $n$-i.e. $\bar{N}:=N \backslash\{n\}$, and define the correspondence 
$D^{k}: I^{N} \rightarrow \mathbb{R}^{N}$ for all $k \in \mathbb{R}_{++}^{\bar{N}}$ as

$$
\begin{aligned}
& D^{k}(S, d):=\{z \in P(S) \mid \text { for all } i \in \bar{N} \text { we have: } \\
&\left.\left(u_{n}(S, d)-z_{n}\right)\left(z_{i}-d_{i}\right)=k_{i}\left(u_{i}(S, d)-z_{i}\right)\left(z_{n}-d_{n}\right)\right\} .
\end{aligned}
$$

It is not hard to verify that $D^{k} \neq D^{k^{\prime}}$ whenever $k, k^{\prime} \in \mathbb{R}_{++}^{\bar{N}}$ with $k \neq k^{\prime}$. The set $\mathcal{D}^{N}:=\left\{D^{k} \mid k \in \mathbb{R}_{++}^{\bar{N}}\right\}$ is defined as the set that contains all such correspondences. For any $k \in \mathbb{R}_{++}^{\bar{N}}$, define

$$
\vartheta^{k}(t):=\left\{z \in G^{k} \mid \sum_{i=1}^{n} z_{i}=t\right\}
$$

where $t \in[1, n]$, and $G^{k}:=\left\{z \in \mathbb{R}_{+}^{N} \mid\left(1-z_{n}\right) z_{i}=k_{i}\left(1-z_{i}\right) z_{n}\right.$ for all $i \in$ $\bar{N}\}$. In Lemma A.1, which can be found in the Appendix, we show that any correspondence $\vartheta^{k}$ is a monotonic curve in $\Theta^{N}$.

Theorem 5.1 For all $k \in \mathbb{R}_{++}^{\bar{N}}$ and $(S, d) \in I^{N}$, we have $D^{k}(S, d)=\left\{\rho^{\vartheta^{k}}(S, d)\right\}$.

It follows from Theorem 5.1 that the set $\mathcal{D}^{N}:=\left\{D^{k} \mid k \in \mathbb{R}_{++}^{\bar{N}}\right\}$ is a subset of the class of individually monotonic bargaining solutions. The following theorem establishes the equivalence between $\left\{\operatorname{Self}^{K S}(., \lambda) \mid \lambda \in \mathbb{R}_{+}^{N}\right\}$ and $\mathcal{D}^{N}$.

Theorem 5.2 For all $(S, d) \in I^{N}$ we have

$$
\operatorname{Self}^{K S}((S, d), \lambda)=D^{k}(S, d)
$$

where $k:=\left(k_{1}, \ldots, k_{n-1}\right)$, and $k_{i}:=\frac{1+\lambda_{n}}{1+\lambda_{i}}$ for all $i \in \bar{N}$. That is,

$\operatorname{Self}^{K S}((S, d), \lambda):=\{z \in P(S) \mid$ for all $i \in \bar{N}$ we have:

$$
\left.\left(1+\lambda_{i}\right)\left(u_{n}(S, d)-z_{n}\right)\left(z_{i}-d_{i}\right)=\left(1+\lambda_{n}\right)\left(u_{i}(S, d)-z_{i}\right)\left(z_{n}-d_{n}\right)\right\} .
$$

With a slight abuse of notation, we henceforth consider $D^{k}(S, d)$ to be an outcome. That is, if $D^{k}(S, d)=\{z\}$, then we write $D^{k}(S, d)=z$. Then also

$$
D^{k}(S, d)=\left(D_{1}^{k}(S, d), \ldots, D_{n}^{k}(S, d)\right) .
$$

From Theorem 5.2 it follows that for each loss aversion profile $\lambda \in \mathbb{R}_{+}^{N}$, we may look at $\operatorname{Self}^{K S}(., \lambda)$ as an asymmetric $n$-player Kalai-Smorodinsky solution where the asymmetry is fully defined by the bargainers' degrees of loss aversion. In the following section we provide an axiomatic characterization of these solutions. 


\section{An Axiomatic Characterization of $\mathcal{D}^{N}$}

From Theorem 5.1 it follows that $\mathcal{D}^{N}$ is a subclass of the individual monotonic bargaining solutions, defined and characterized by Peters and Tijs (1984). Of their axioms we retain Scale Invariance [SI] and Individual Monotonicity [IM].

(SI) $\varphi: B^{N} \rightarrow \mathbb{R}^{n}$ satisfies Scale Invariance if $t(\varphi(S, d))=\varphi(t(S), t(d))$, where $t: \mathbb{R}^{n} \rightarrow \mathbb{R}^{n}$ is a linear transformation $t(x):=\alpha+\beta x$, with $\alpha \in \mathbb{R}^{N}$, $\beta \in \mathbb{R}_{++}^{n}$, and $t(S):=\alpha+\beta S$ for $S \subset \mathbb{R}^{N}$.

(IM) $\varphi: B^{N} \rightarrow \mathbb{R}^{n}$ satisfies Individual Monotonicity if $\varphi_{i}(S, d) \leq \varphi_{i}(T, d)$ for all $(S, d),(T, d) \in B^{N}$ and $i \in N$ with $S \subseteq T$ and $u_{j}(S)=u_{j}(T)$ for all $j \in N \backslash\{i\}$.

The axiom of SI is consistent with the premise that bargainers' preferences are representable by von Neumann-Morgenstern utility functions. Kalai and Smorodinsky (1975) introduced IM as a possible alternative to Nash's (1950) Independence of Irrelevant Alternatives after this axiom had been criticized. A third well-known property we require is Strong Individual Rationality [SIR].

(SIR) $\varphi: B^{N} \rightarrow \mathbb{R}^{n}$ satisfies Strong Individual Rationality if $\varphi(S, d)>d$ for all $(S, d) \in B^{N}$.

We now introduce a novel axiom called Proportional Concession Invariance [PCI]. One can consider a solution outcome $\varphi(S, d)$ as a representation of concessions that bargainers make with respect to their utopia point values $u(S, d)$. The PCI axiom then says that if we replace $u(S, d)$ by a point $\hat{u}$ on the line segment connecting $\varphi(S, d)$ and $u(S, d)$, and shrink the bargaining set accordingly, then the solution outcome should not change. In other words, if the players' utopia values are reduced in such a way that their concessions with respect to the original solution outcome change proportionally, then this solution outcome should not change.

(PCI) $\varphi: B^{N} \rightarrow \mathbb{R}^{n}$ satisfies Proportional Concession Invariance if for a bargaining problem $(S, d) \in B^{N}$ with solution $\varphi(S, d)$, and a bargaining problem $(\hat{S}, d)$ with

$$
\hat{S}:=\{z \in S \mid z \leq \hat{u}\},
$$

where $\hat{u}=\alpha \varphi(S, d)+(1-\alpha) u(S, d)$ for some $\alpha \in[0,1]$, we have $\varphi(\hat{S}, d)=$ $\varphi(S, d)$.

The property PCI can also be thought of as a very weak form of IIA, and is therefore satisfied by the Nash bargaining solution and its asymmetric variants. Furthermore, observe that PCI is the counterpart to Disagreement Point Convexity [DPC], one of the axioms used by Peters and van Damme (1991) to characterize the class of asymmetric Nash bargaining solutions. This property requires that the solution outcome $\varphi(S, d)$ remain unchanged if we replace $d$ 
by a point $\hat{d}$ on the line segment connecting the disagreement point $d$ and the solution outcome $\varphi(S, d)$.

It is worth noting that the characterization of $\mathcal{D}^{N}$ makes no use of Pareto Optimality [PO]. In fact, in the Appendix, we use an argument similar as in Roth (1977) to show that PO is implied by our axioms. The following theorem states our characterization result.

Theorem 6.1 Let $\varphi: I^{N} \rightarrow \mathbb{R}^{N}$ be a bargaining solution. Then $\varphi \in \mathcal{D}^{N}$ if and only if $\varphi$ satisfies SIR, SI, IM, and PCI.

\section{Concluding Remarks}

We have established that bargaining games with $n$ possibly loss averse players, $n \geq 2$, have exactly one self-supporting outcome under the Kalai-Smorodinsky solution. Moreover, we defined an asymmetric $n$-player version of the KS solution which directly captures the asymmetry resulting from the bargainers' degrees of loss aversion. We may summarize these results in the following corollary.

Corollary 7.1 Let $\lambda \in \mathbb{R}_{+}^{N}$ be the profile of loss aversion. For every $(S, d) \in$ $I^{N}$, an outcome $z \in S$ is self-supporting under the KS solution if and only if $z=D^{k}(S, d)$, where $k_{i}:=\frac{1+\lambda_{n}}{1+\lambda_{i}}$ for all $i \in \bar{N}$.

We have characterized the class $\mathcal{D}^{N}$ of all bargaining solutions $D^{k}$ by the axioms of Strong Individual Rationality, Scale Invariance, Individual Monotonicity, and Proportional Concession Invariance. While the first three properties are standard in the axiomatic bargining literature, the last one is newly defined in this article. 


\section{A Collected Proofs}

In this appendix we prove the claims made in the article.

\section{A.1 The proof of Lemma 3.1}

Let $(S, d) \in B^{N}, \lambda \in \mathbb{R}_{+}^{N}$, and $r \in \mathbb{R}^{N}$. Since the Shalev transformation is continuous, one-to-one, and preserves the ordering of payoffs, we have that

- $w(S, \lambda, r)$ is non-empty, closed, and comprehensive,

- $w(d, \lambda, r) \in w(S, \lambda, r)$,

- there are $z \in w(S, \lambda, r)$ with $z>w(d, \lambda, r)$, and

- $w\left(S_{d}, \lambda, r\right)$ is bounded.

Left to show is that $w(S, \lambda, r)$ is convex. Let $x, y \in S$ and $t \in[0,1]$. By convexity of $S$, we have $t x+(1-t) y \in S$. By the fact that $w$ is concave in the first coordinate, we have

$$
w(t x+(1-t) y, \lambda, r) \geq t w(x, \lambda, r)+(1-t) w(y, \lambda, r) .
$$

By comprehensiveness of $w(S, \lambda, r)$ this implies

$$
t w(x, \lambda, r)+(1-t) w(y, \lambda, r) \in w(S, \lambda, r) .
$$

This implies convexity of $w(S, \lambda, r)$.

\section{A.2 The proof of Theorem 5.1}

First, we show the following.

Lemma A.1 Let $k \in \mathbb{R}_{++}^{\bar{N}}$. Then the correspondence $\vartheta^{k}(t), t \in[1, n]$, is a monotonic curve.

Proof. Let $\bar{s} \in[1, n]$. We show that there is a unique point $z^{*} \in \mathbb{R}_{+}^{N}$ such that $\vartheta^{k}(\bar{s})=z^{*}$. That is, we show that the system of equations

$$
\begin{aligned}
& \left(1-z_{n}\right) z_{i}=k_{i}\left(1-z_{i}\right) z_{n} \text { for all } i \in \bar{N} \\
& \sum_{j=1}^{n} z_{j}=\bar{s},
\end{aligned}
$$

has exactly one solution $z^{*}$ in $\mathbb{R}_{+}^{N}$. Suppose the system has a solution $z \in \mathbb{R}_{+}^{N}$, and suppose $z_{n}>1$. Then for each $i \in \bar{N}$ we either have $z_{i}>1$ or $z_{i}<0$, which by $z \in \mathbb{R}_{+}^{N}$ implies $z_{i}>1$ for all $i \in N$. Since this is a violation of (5), we must have $z_{n} \leq 1$. Since $\left(1-k_{i}\right)^{-1} \notin[0,1]$ for all $i \in \bar{N}$, we can write

$$
z_{i}=\frac{k_{i} z_{n}}{1-z_{n}\left(1-k_{i}\right)} \text { for all } i \in \bar{N} \text {. }
$$


Then for all $i \in \bar{N}, z_{i}$ is strictly increasing in $z_{n}$ on the domain $[0,1]$. The observations that

$$
\begin{array}{ll}
\sum_{j=1}^{n} z_{j}=0 \leq \bar{s} & \text { for } z_{n}=0, \text { and } \\
\sum_{j=1}^{n} z_{j}=n \geq \bar{s} & \text { for } z_{n}=1,
\end{array}
$$

together with continuity of $\sum_{j=1}^{n} z_{j}$ in $z_{n}$ then imply that there is exactly one $z^{*} \in \mathbb{R}_{+}^{N}$ that solves the system of equations (4) and (5). It follows that the correspondence $\vartheta^{k}$ is a map. Moreover, for all $1 \leq s \leq t \leq n$ we have $0<$ $\vartheta^{k}(s) \leq \vartheta^{k}(t) \leq e^{N}$ and $\sum_{i \in N} \vartheta_{i}^{k}(s)=s$. It follows that $\vartheta^{k}$ is a monotonic curve.

To prove Theorem 5.1, we show that each map $D^{k} \in \mathcal{D}^{N}$ is the bargaining solution associated with the monotonic curve $\vartheta^{k}$ as defined in (3).

Proof of Theorem 5.1. Consider a normalized bargaining problem $T \in I_{0}^{N}$, i.e. $u(T)=e^{N}$. Let $k \in \mathbb{R}_{++}^{\bar{N}}$ and observe that by (2) we have

$$
D^{k}(T)=P(T) \cap G^{k} .
$$

By convexity of $T$ we have $P(T) \subset\left\{z \in \mathbb{R}_{+}^{N} \mid \sum_{j \in N} z_{j} \geq 1\right\}$, implying

$$
D^{k}(T)=P(T) \cap\left\{\vartheta^{k}(t) \mid t \in[1, n]\right\},
$$

where $\vartheta^{k}$ is defined by (3). It follows that $D^{k}(T)=\left\{\rho^{\vartheta^{k}}(T)\right\}$. From this it is easily established that

$$
D^{k}(S, d)=\left\{\rho^{\vartheta^{k}}(S, d)\right\}
$$

for all $(S, d) \in I^{N}$.

\section{A.3 The Proof of Theorem 5.2}

We start with the following lemma.

Lemma A.2 Let $(S, d) \in I^{N}$. Then $D^{k}(S, d)=K S(S, d)$ if and only if $k=e^{\bar{N}}$.

Proof. It is easy to show that for any $t \in[1, n)$, we have $\vartheta^{k}(t)=\hat{\vartheta}(t)$ if and only if $k=e^{\bar{N}}$. The result then follows from Theorem 5.1 and the definition of $K S$.

Proof of Theorem 5.2 Let $(S, d) \in I^{N}$ and $\lambda \in \mathbb{R}_{+}^{N}$, and let $u \equiv u(S, d)$. By Lemma A.2 and the fact that $K S(S, d) \geq d$, we have

$$
\begin{aligned}
& K S(w((S, d), \lambda, z))=\{x \in P(w(S, \lambda, z)) \mid \text { for all } i \in \bar{N} \text { we have } \\
& \left.\quad\left(u_{n}-x_{n}\right)\left(x_{i}-\left(1+\lambda_{i}\right) d_{i}+\lambda_{i} z_{i}\right)=\left(u_{i}-x_{i}\right)\left(x_{n}-\left(1+\lambda_{n}\right) d_{n}+\lambda_{n} z_{n}\right)\right\} .
\end{aligned}
$$


Observe that $z \in \operatorname{Self}^{K S}((S, d), \lambda)$ if and only if $z=K S(w((S, d), \lambda, z))$. That is, $z \in \operatorname{Self}^{K S}((S, d), \lambda)$ iff

(i) $z \in P(w(S, \lambda, z))$, and

(ii) $\left(u_{n}-z_{n}\right)\left(z_{i}-\left(1+\lambda_{i}\right) d_{i}+\lambda_{i} z_{i}\right)=\left(u_{i}-z_{i}\right)\left(z_{n}-\left(1+\lambda_{n}\right) d_{n}+\lambda_{n} z_{n}\right)$ for all $i \in \bar{N}$

From $z=w(z, \lambda, z), P(w(S, \lambda, z))=w(P(S), \lambda, z)$, and the fact that the Shalev transformation is one-to-one, it follows that $z \in P(w(S, \lambda, z))$ is equivalent to $z \in P(S)$.

Define $k:=\left(k_{1}, \ldots, k_{n-1}\right)$ where $k_{i}:=\frac{1+\lambda_{n}}{1+\lambda_{i}}$ for all $i \in \bar{N}$. Observe that the statement in (ii) is equivalent to

$$
\left(u_{n}-z_{n}\right)\left(z_{i}-d_{i}\right)=k_{i}\left(u_{i}-z_{i}\right)\left(z_{n}-d_{n}\right) \text { for all } i \in \bar{N} .
$$

It follows that $z \in \operatorname{Self}^{K S}((S, d), \lambda)$ is equivalent to

$$
z \in\left\{x \in P(S) \mid\left(u_{n}-x_{n}\right)\left(x_{i}-d_{i}\right)=k_{i}\left(u_{i}-x_{i}\right)\left(x_{n}-d_{n}\right) \text { for all } i \in \bar{N}\right\} .
$$

Hence, $\operatorname{Self} f^{K S}((S, d), \lambda)=D^{k}(S, d)$.

\section{A.4 The proof of Theorem 6.1}

The standard axiom of Pareto Optimality is useful for the proof.

$(\mathrm{PO}) \varphi: B^{N} \rightarrow \mathbb{R}^{N}$ satisfies PO if $\varphi(S, d) \in P(S)$ for all $(S, d) \in B^{N}$.

From Peters and Tijs (1984) we obtain the following.

Lemma A.3 (Peters and Tijs, 1984) Let $\varphi: I^{N} \rightarrow \mathbb{R}^{N}$ be a bargaining solution. Then $\varphi$ satisfies $P O, S I$, and $I M$, if and only if $\varphi=\rho^{\vartheta}$ for some $\vartheta \in \Theta^{N}$.

This we use to establish the following result.

Proposition A.4 Let $\varphi: I^{N} \rightarrow \mathbb{R}^{N}$ be a bargaining solution in $\mathcal{D}^{N}$. Then $\varphi$ satisfies SIR, SI, IM, and PCI.

Proof. Since $\varphi \in \mathcal{D}^{N}$ we have $\varphi=D^{k}$ for some $k \in \mathbb{R}_{++}^{\bar{N}}$. By Theorem 5.1, we have $\varphi=\rho^{\vartheta}$ where $\vartheta \in \Theta^{N}$, which by Lemma A.3 implies that $\varphi$ satisfies SI and IM.

Consider a bargaining problem $(S, d) \in I^{N}$, and the solution $\varphi(S, d)=: z$. To see that $\varphi$ satisfies $\mathrm{SIR}$, suppose there is an $i \in N$ such that $z_{i}=d_{i}$. Observe that $z \in P(S)$ and

$$
\left(u_{n}(S, d)-z_{n}\right)\left(z_{j}-d_{j}\right)=k_{j}\left(u_{j}(S, d)-z_{j}\right)\left(z_{n}-d_{n}\right) \text { for all } j \in \bar{N} .
$$

If $i=n$, then $z_{j}=d_{j}$ for all $j \in \bar{N}$, implying $z=d$. Let $i \in \bar{N}$, and observe that $z_{i}=d_{i}$ implies $z_{n}=d_{n}$, and thus $z=d$. Since $d \notin P(S)$, we arrive at a contradiction. It follows that $z>d$. 
To see that $\varphi$ satisfies PCI, consider the problem $(\hat{S}, d) \in I^{N}$ where

$$
\hat{S}:=\{x \in S \mid x \leq \hat{u}\},
$$

with $\hat{u}=\alpha z+(1-\alpha) u(S, d)$ for some $\alpha \in(0,1)$. Then $\hat{u}-z=(1-\alpha)(u(S, d)-z)$, implying that

$$
\left(\hat{u}_{n}-z_{n}\right)\left(z_{i}-d_{i}\right)=k_{i}\left(\hat{u}_{i}-z_{i}\right)\left(z_{n}-d_{n}\right) \text { for all } i \in \bar{N} .
$$

Since $z \in \hat{S}$ and $z \in P(S)$, we have $z \in P(\hat{S})$. This and (6) together imply $D^{k}(\hat{S}, d)=z$. Hence, $\varphi$ satisfies PCI.

For the reverse implication we require two additional lemmas.

Lemma A.5 Let $(S, d) \in I^{N}$, and $z \in S \backslash P(S)$. Then for the function $f$ : $[0,1] \rightarrow \mathbb{R}^{N}$ defined as

$$
f(\alpha):=(1-\alpha) z+\alpha u(S, d),
$$

there is exactly one $\alpha^{*} \in[0,1]$ such that $f\left(\alpha^{*}\right) \in P(S)$.

Proof. By compactness of $S$ we have that

$$
\alpha^{*}:=\max \{\alpha \mid f(\alpha) \in S\}
$$

is well-defined. We now show that $f\left(\alpha^{*}\right) \in P(S)$. Suppose $f\left(\alpha^{*}\right) \notin P(S)$. By condition (1), it follows that for each $i \in N$ with $z_{i}<u_{i}(S, d)$, there is an $\varepsilon_{i}>0$ such that $f\left(\alpha^{*}\right)+\varepsilon_{i} e^{i} \in S$. Then by convexity of $S$ there is an $\varepsilon>0$, such that

$$
f\left(\alpha^{*}\right)+\varepsilon(u(S, d)-z) \in S .
$$

But then there is a $\beta>\alpha^{*}$ with $f(\beta) \in S$. This is a contradiction. To show uniqueness, let $\alpha_{1}, \alpha_{2} \in[0,1]$ with $\alpha_{1} \neq \alpha_{2}$, and suppose $f\left(\alpha_{1}\right), f\left(\alpha_{2}\right) \in$ $P(S)$. Without loss of generality, assume $\alpha_{2}>\alpha_{1}$. Then since $u(S, d) \geq z$ and $u(S, d) \neq z$, we have $f\left(\alpha_{2}\right) \geq f\left(\alpha_{1}\right)$ and $f\left(\alpha_{2}\right) \neq f\left(\alpha_{1}\right)$. Since $f\left(\alpha_{1}\right) \in P(S)$, this implies $f\left(\alpha_{2}\right) \notin S$, a contradiction.

Lemma A.6 Let $\varphi: I^{N} \rightarrow \mathbb{R}^{N}$ be a solution satisfying SIR, SI, and PCI. Then $\varphi$ satisfies $P O$.

Proof. Let $\varphi: I^{N} \rightarrow \mathbb{R}^{N}$ be a bargaining solution satisfying SIR, SI, and PCI. By SI it is sufficient to restrict attention to bargaining problems in $I_{0}^{N}$. Let $S \in I_{0}^{N}$. By SIR we have $\varphi(S)>0$. Now assume $\varphi(S) \notin P(S)$. By Lemma A.5, there is a single $z^{*} \in P(S)$, such that

$$
z^{*}=(1-\alpha) \varphi(S)+\alpha u(S) \text { for some } \alpha \in(0,1] .
$$

Define $\hat{S}:=\left\{x \in S \mid x \leq z^{*}\right\}$, and observe that by PCI we have

$$
\varphi(\hat{S})=\varphi(S) .
$$


Similarly, for the set $T:=\{x \in S \mid x \leq \varphi(S)\}$ we have $\varphi(T)=\varphi(S)$. Now observe that $\hat{S}=\left[z^{*}(\varphi(S))^{-1}\right] T$. Then by SI we have

$$
\varphi(\hat{S})=z^{*}(\varphi(S))^{-1} \varphi(T)=z^{*}(\varphi(S))^{-1} \varphi(S)=z^{*} .
$$

This contradicts $\varphi(\hat{S})=\varphi(S)$.

Proposition A.7 Let $\varphi: I^{N} \rightarrow \mathbb{R}^{N}$ be a bargaining solution satisfying SIR, SI, IM, and PCI. Then $\varphi \in \mathcal{D}^{N}$.

Proof. Since $\varphi$ satisfies SIR, SI, and PCI, it follows from Lemma A.6 that $\varphi$ satisfies PO. Then by Lemma A.3 it follows that $\varphi \equiv \rho^{\vartheta^{*}}$ for some monotonic curve $\vartheta^{*} \in \Theta^{N}$.

Consider the problem

$$
H:=\operatorname{Conv}\left(\left\{e^{i} \mid i \in N\right\} \cup\{0\}\right),
$$

and observe that $H \in I_{0}^{N}$. If there is an $i \in N$ with $\varphi_{i}(H)=1$, then $\varphi_{j}(H)=0$ for all $j \neq i$, which is a violation SIR. Hence, $0<\varphi(H)<e^{N}$. Hence, $k:=$ $\left(k_{1}, \ldots, k_{n-1}\right)$, where

$$
k_{i}:=\frac{1-\varphi_{n}(H)}{1-\varphi_{i}(H)} \cdot \frac{\varphi_{i}(H)}{\varphi_{n}(H)},
$$

is well-defined and $k \in \mathbb{R}_{++}^{\bar{N}}$.

In what follows, we show that

$$
\vartheta^{*}(t)=\vartheta^{k}(t)
$$

for all $1 \leq t \leq n$. We do this in three steps. Let $\bar{t} \in[1, n]$. Then

1. we construct a specific problem $S \in I_{0}^{N}$,

2. we show that $\varphi(S)=\vartheta^{k}(\bar{t})$, and

3. we show that $\varphi(S)=\vartheta^{*}(\bar{t})$.

By (8) we have $\rho^{\vartheta^{k}}=\rho^{\vartheta^{*}}$, which together with $\varphi=\rho^{\vartheta^{*}}$ and $D^{k}=\rho^{\vartheta^{k}}$ establishes $\varphi=D^{k}$.

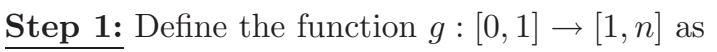

$$
g(\alpha):=\sum_{i \in N} \frac{\varphi_{i}(H)}{\alpha \varphi_{i}(H)+(1-\alpha)} .
$$

From the fact that $g$ is strictly increasing ${ }^{2}$ and continuous, and the fact that $g(0)=1$ and $g(1)=n$, it follows that for each $t \in[1, n]$ there is a unique $\alpha \in[0,1]$ such that $g(\alpha)=t$.

\footnotetext{
${ }^{2}$ This follows from $\varphi(H) \neq 0$ and $\varphi(H) \neq e^{N}$.
} 
Let $\beta:=\bar{\alpha} \varphi(H)+(1-\bar{\alpha}) e^{N}$, where $\bar{\alpha}$ is such that $g(\bar{\alpha})=\bar{t}$. Then define the problem $S \in I_{0}^{N}$ as

$$
S:=\left\{\beta^{-1} z \mid z \in H \text { and } z \leq \beta\right\} .
$$

Since $\varphi$ satisfies SI and PCI, we have

$$
\varphi(S)=\beta^{-1} \varphi(H) .
$$

It follows from (10) and (9), and the fact that $g(\bar{\alpha})=\bar{t}$ that $\sum_{i \in N} \varphi_{i}(S)=\bar{t}$.

Step 2: Rewriting (7) yields

$$
\left(1-\varphi_{n}(H)\right) \varphi_{i}(H)=k_{i}\left(1-\varphi_{i}(H)\right) \varphi_{n}(H) \text { for all } i \in \bar{N} .
$$

From the definition of $\beta$ we have $\beta-\varphi(H)=(1-\bar{\alpha})\left(e^{N}-\varphi(H)\right)$. Thus,

$$
\left(\beta_{n}-\varphi_{n}(H)\right) \varphi_{i}(H)=k_{i}\left(\beta_{i}-\varphi_{i}(H)\right) \varphi_{n}(H) \text { for all } i \in \bar{N} .
$$

For each $i \in \bar{N}$, we can multiply both sides of the equation by $\frac{1}{\beta_{n} \beta_{i}}$. By (10) this yields

$$
\left(1-\varphi_{n}(S)\right) \varphi_{i}(S)=k_{i}\left(1-\varphi_{i}(S)\right) \varphi_{n}(S) \text { for all } i \in \bar{N} .
$$

It follows that $\varphi(S) \in G^{k}$. Since $\sum_{i \in N} \varphi_{i}(S)=\bar{t}$, we have

$$
\varphi(S)=\vartheta^{k}(\bar{t})
$$

Step 3: Since $\varphi \equiv \rho^{\vartheta^{*}}$, and $S \in I_{0}^{N}$ with $u(S)=e^{N}$, we have

$$
\{\varphi(S)\}=P(S) \cap\left\{\vartheta^{*}(t) \mid t \in[1, n]\right\},
$$

which implies $\varphi(S)=\vartheta^{*}\left(t^{*}\right)$ for some $t^{*} \in[1, n]$. From the definition of monotonic curves we obtain

$$
t^{*}=\sum_{i \in N} \vartheta_{i}^{*}\left(t^{*}\right)=\sum_{i \in N} \varphi_{i}(S)=\bar{t}
$$

Hence,

$$
\varphi(S)=\vartheta^{*}(\bar{t})
$$

Combining (11) and (12) yields the desired result.

Theorem 6.1 is then established by Propositions A.4 and A.7. 


\section{References}

[1] Kahneman D., and A. Tversky. Prospect theory: an analysis of choice under risk. Econometrica 47(2):263-291, 1979.

[2] Kahneman D., and A. Tversky. Choices, Values, and Frames. American Psychologist 39(4):341-350, 1984.

[3] Kalai E., and M. Smorodinsky. Other solutions to Nash's bargaining problem. Econometrica, 43(3):513-518, 1975.

[4] Kannai Y. Concavifiability and constructions of concave utility functions. Journal of mathematical Economics, 4(1):1-56, 1977.

[5] Köbberling V., and H. Peters. The effect of decision weights in bargaining problems. Journal of Economic Theory, 110(4):154-175, 2003.

[6] Nash J. The bargaining problem. Econometrica, 18(2):155-162, 1950.

[7] Peters H., and E. van Damme. Characterizing the Nash and Raiffa bargaining solutions by disagreement point axioms. Mathematics of Operations Research, 16(3):447-461, 1991.

[8] Peters H., and S. Tijs. Individually monotonic bargaining solutions for n-person games. Methods of Operations Research, 51:377-384, 1984.

[9] Raiffa H. Arbitration schemes for generalized two-person games, in: Contributions to the Theory of Games II. Kuhn H.W., and A.W. Tucker (eds.) Annals of Mathematics Studies, 28:361-387, 1953.

[10] Roth A.E. An impossibility result concerning $n$-person bargaining games. International Journal of Game Theory, 8(3):129-132, 1979.

[11] Roth A.E. Individual rationality and Nash's solution to the bargaining problem. Mathematics of Operations Research, 2(1):64-65, 1977.

[12] Shalev J. Loss aversion equilibrium. International Journal of Game theory, 29(2):269-287, 2000.

[13] Shalev J. Loss aversion and bargaining. Theory and Decision, 52(3):201232,2002 . 